ORIGINAL PAPER

\title{
Surgical Treatment of Congenital High Condition of the Shoulder Blade in Children
}

\author{
Akhror Makhmudovitch JURAEV', Feruza Shavkatovna ALIMUKHAMEDOVA
}

\begin{abstract}
The purpose of our paper is to study the results of surgical treatment of patients with congenital high standing of the scapula. Congenital high standing of the scapula, Sprengel disease is a complex combination of anomalies in the development of the shoulder girdle, spine and chest. The main clinical manifestations of the pathology are the high standing of the shoulder blade of the abduction restriction in the shoulder joint and a pronounced cosmetic defect. The results of surgical treatment of patients with congenital high standing of the scapula, taking into account the form and severity of the disease were studied. The study was conducted at the Department of Pediatric Orthopedics of RSSPMCTO. 85 children aged 3 to 14 years with congenital high standing of the scapula were treated for the period from 2005 to 2018, there were 26 (31\%) boys and girls - 59 (69\%). Patients with congenital high position of the scapula underwent Ternovsky 11 (13\%) and Pozdeev 74 (87\%) surgery. After the operation, the thoracobrachial cast were put on patients for 4 weeks. In addition, it was prescribed the physiotherapy exercises on the fingers and UHF on top of the plaster cast. Long-term results showed that out of 85 (100\%) patients 73 (86\%) were good and $12(14 \%)$ were satisfactory. Evaluation of treatment results was carried out according to cosmetic and functional parameters. Our research conclusions show that the operation according to the Pozdeev method is atraumatic and it is the method of choice for surgical treatment of Sprengel disease in children.
\end{abstract}

Keywords: surgery, congenital, scapula, children, shoulder.

${ }^{1}$ GU Republican Specialized Scientific and Practical Medical Center of Traumatology and Orthopedics, Tashkent, Uzbekistan
Corresponding author.

Feruza Shavkatovna ALIMUKHAMEDOVA, GU Republican Specialized Scientific and Practical Medical Center of Traumatology and Orthopedics, Tashkent, Uzbekistan. E-mail: Feruzaash@mail.ru 


\section{INTRODUCTION}

The high standing of the scapula is a complex of developmental anomalies, the main manifestations of which are: violation of spatial position, growth disorders and deformation of the scapula, displacement in the cranial direction of the distal end of the clavicle, hypoplasia of the muscles of the shoulder girdle and trunk, up to their full aplasia, impaired functional state of the neuromuscular apparatus, pathology of the main vessels and impaired peripheral blood flow.

The relevance of the problem is due to the complexity of the treatment of pathology. Hypoplasia, aplasia of the trapezius, rhomboid, supraspinatus, hypodermic, deltoid, anterior dentate muscles and the muscle that raises the shoulder blade accompanying severe muscle and bone forms of pathologies are significantly exacerbate the course and complicate the prognosis of the disease $^{1}$. To date, there is no single view on the cause of the development of Sprengel disease, the results of treatment are contradictory.

Aim of study is to study the results of surgical treatment of patients with congenital high standing of the scapula according to the stage and severity of the disease.

\section{MATERIAL AND METHODS}

This study was conducted in the Department of Pediatric Orthopedics RSSPMCTO. Between 2005 and 2018 the 85 children were treated. There were aged from 3 to 14 years old, 26 (31\%) boys and 59 (69\%) girls. The deformation of the on the side of localization were divided as following: left-sided in $36(45 \%)$ cases, right-sided in $32(41 \%)$ cases and bilateral in $8(14 \%)$ cases. Besides, concerning to the severe of lesion it was divided as follows: mild-9, soft-tissue medium-28, soft-tissue heavy-23, bone medium-15, bone heavy- 10 .

Children with a muscle form of mild severity of the disease are subject to conservative treatment. To improve trophism and muscle functions of the shoulder girdle and trunk a complex of physiotherapy exercises is prescribed. Physiotherapeutic treatment is carried out aimed at improving the functional state of the vascular and nervous systems (electrophoresis with aminophylline, pentoxifylline in the collar region), as well as electrical stimulation of the muscles involved in the shoulder abduction. It was recommended swimming and health-resort treatment.

The indicators for surgical treatment are moderate and severe diseases in children older than 3 years. Surgical treatment of children with Sprengel disease is performed according to the Pozdeev method ${ }^{2}$. Moreover, during the surgery to children with bone and soft tissue forms of moderate and severe A degree of the disease an osteotomy of the coracoid appendage is performed. By turning in the acromioclavicular joint the scapula is relegated. The transverse osteotomy of the inner and osteoclasia of an outer cortical plastic scapula is performed, which adapts the contacting surfaces of the scapula and chest.
Figure 1. Appearance of the patient's back K. 6 years old, with congenital high standing of the scapula on the right II degree.

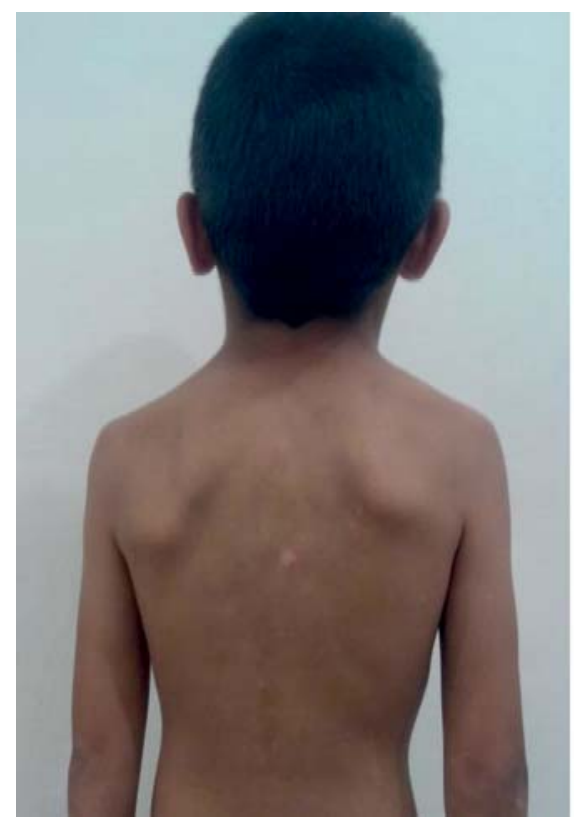

Figure 2. Computed tomography of the shoulder blades of the same patient, which determines the high standing of the shoulder blades on the right II degree. 
Surgical treatment of children with soft tissue and bone forms of diseases of severe B degree. It is performed an osteotomy of the coracoid appendage. A pocket is formed between the chest and the latissimus spine muscle. The scapula is relegated up to the level of a health one and its low-level is fixed in the formed packet with three $\mathrm{P}$ shaped stitches to the widest muscle, and they are replaced by paravertebral muscles.

The shortening of the clavicle is performed by a resection of the part in which the fragments overlapping is occurred one after another. The osteosynthesis of clavicle fragments with Kirschner pin is performed retrogradely.

The immobilization of shoulder girdle at the osteotomy of the clavicle is performed with SmirnovWeinstein cast bandage for 6 weeks. Various methods are used to eliminate cosmetic defects and restore the function of upper limb depending on type of a disease. The result of treatment in all forms is fixed after the surgery with a complex of rehabilitation therapy.

In the postoperative period, to the patients with congenital high standing of the scapula it is recommended a rehabilitation treatment, including immo- bilization of the cervical spine with the tilted of head to the healthy side, physical therapy, physiotherapeutic and drug treatment aimed at restoring the movements of the upper limb in the shoulder joint, increasing tone and restoration of muscle functions, scar resorption tissues, improving regional blood flow and trophic nervous system. Clinical observation of patients is carried out until the end of their growth.

Indications for surgical treatment were moderate and severe disease in children older than 3 years. The main principles of restoration of a cosmetic defect of the scapula were:

1. The mobilization of the scapula, namely its medial edge, the upper mesial and lower angles, the anterior surface of the scapula;

2. Elimination of pathological rotation of the scapula;

3. Relegation of the scapula to the level of healthy one;

4. Adaptation of the anterior surface of the scapula to the chest.

The restoration of the function of the upper limb was carried out by the following methods:
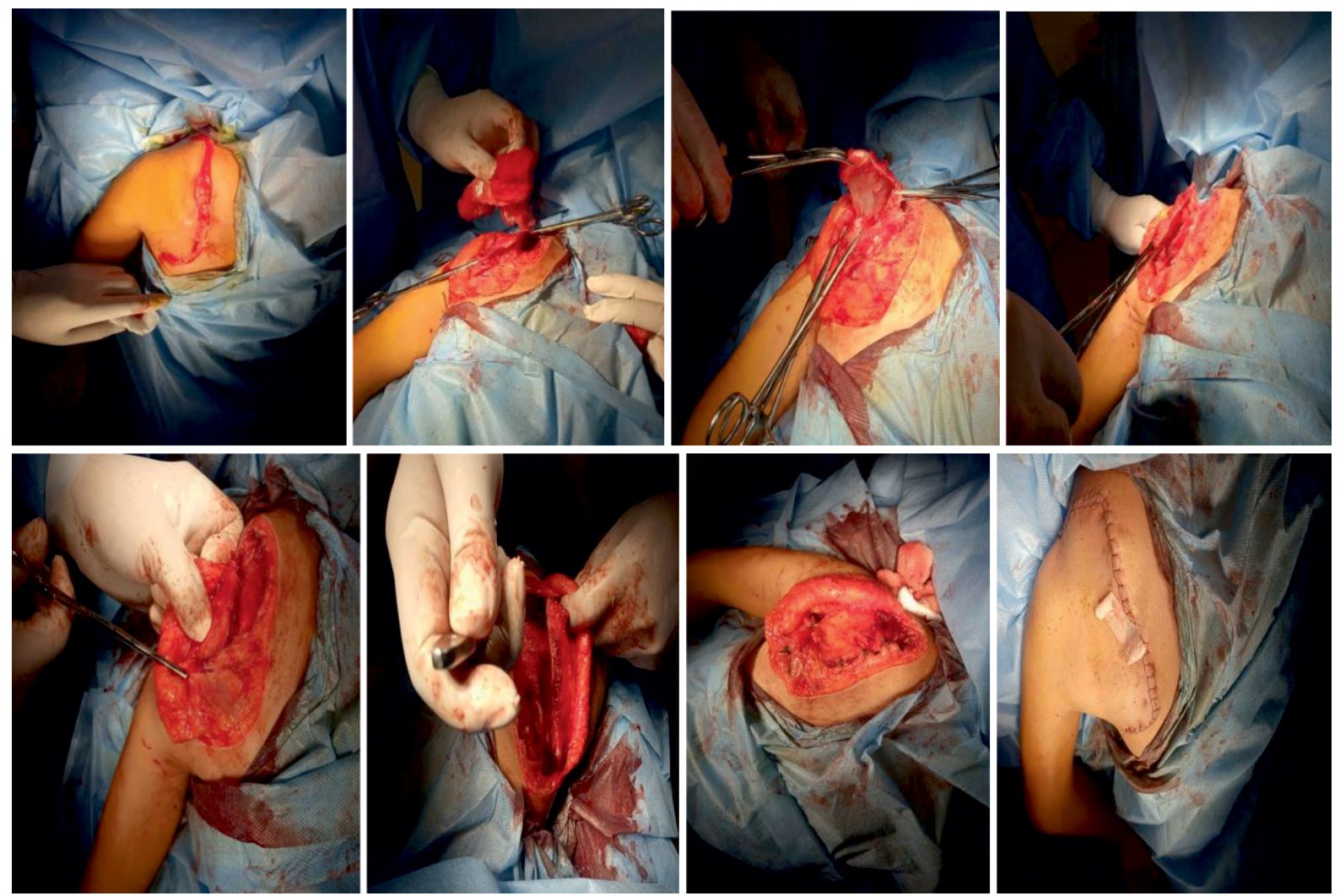

Figure 3. Photo of the operation process of patient N. 5 years old, with congenital high standing of the shoulder blade on the left. 
1. Mobilization of the suprascapular neurovascular bundle. For this purpose, a semicircular osteotomy of the transverse scapular incision was performed. This technique made it possible to make the suprascapular neurovascular bundle mobile and prevented the disinnervation of the supraspinatus and infraspinatus muscles during the reduction of the scapula.

2. The physiological fixation of the scapula was carried out by suturing its lower angle in the "pocket" between the chest and the latissimus dorsi.

The elimination of a cosmetic defect and restoration of the function of the upper limb was carried out as follows:

Surgical technique. A narcosis is endotracheal. The position of the patient is on the abdomen with a roller placed under the shoulders. It is made a skin incision that borders the upper and medial edges of the scapula, up to level VII of the rib. Skin-subcutaneous-fascial flaps: lateral - to the upper and outer edges of the scapula and its lower corner; medial - to the spinous processes of the vertebrae is mobilized. The trapezius and rhomboid muscles are cut off from the medial edge of the scapula, a fibrous cord is dissected. The muscle that lifts the scapula is crossed at the point of attachment to the scapula. Along the medial edge of the scapula, along its anterior surface, muscle fibers of the anterior dentate and subscapularis are cut off. Soft tissue is separated from the anterior surface of the scapula to the transverse notch, preserving the periosteum as much as possible. A subperiosteal transverse incision of the scapula is distinguished, an osteotomy of the scapular bone is made with a grooved chisel, departing from the transverse incision of the scapula 3-5 $\mathrm{mm}$. Subperiosteally, osteotomy of the base of the coracoid process is isolated and produced, while the bit is directed from the bottom up and from the middle outwards. By turning in the acromioclavicular joint, the pathological rotation of the scapula is eliminated and its reduction is performed (the reference is the spine of the scapular bones, and in case of bilateral damage, the physiological norm, the lower edge of the second thoracic vertebra).

The congruence of the sliding surfaces of the chest and scapula is assessed. With severe deformation of the latter, an osteotomy of the inner cortical plate and osteoclasia of the outer at the top of the deformity of the scapula are produced.

In the gap between the chest and the latissimus dorsi, a "pocket” is formed, where the lower corner of the scapula is immersed. The shoulder blade is fixed in a new place by stitching its lower corner in the formed „pocket" to the latissimus dorsi muscle with 3 to $4 \mathrm{U}$ shaped sutures. In the position of the achieved correction, the trapezoid, rhomboid muscles are fixed with U-shaped sutures.

The wound is sutured in layers. Wound drainage is performed in the upper and lower divisions by two rubber tapes. In the postoperative period, the limb was immobilized with a kerchief bandage for 10-14 days.

In the postoperative period, a rehabilitation treatment, including immobilization of the cervical spine with the head tilted to the healthy side, physical therapy, physiotherapeutic and drug treatment aimed at restoring the movements of the upper limb in the shoulder joint, increasing tone and restoration of muscle functions, scar resorption tissues, improving regional blood flow and trophic nervous system are required for the patients with congenital high standing of the scapula. Clinical observation of patients is carried out until the end of their growth.

At a severe degree of deformation, the shoulder joint with the scapula is raised upward and displaced anteriorly and the scapula is reduced in size and is located above the healthy one by more than $5 \mathrm{~cm}$ to $10 \mathrm{~cm}$. The muscles are strongly hypotrophic and the abduction does not exceed 120-90 degrees.

Observations showed that a severe degree of the disease in 10 patients was combined with an additional ometebral bone, and in 6 patients it was combined with Klippel-Feil disease. In addition, various degrees of rib deformation and scoliosis were found in the remaining patients.

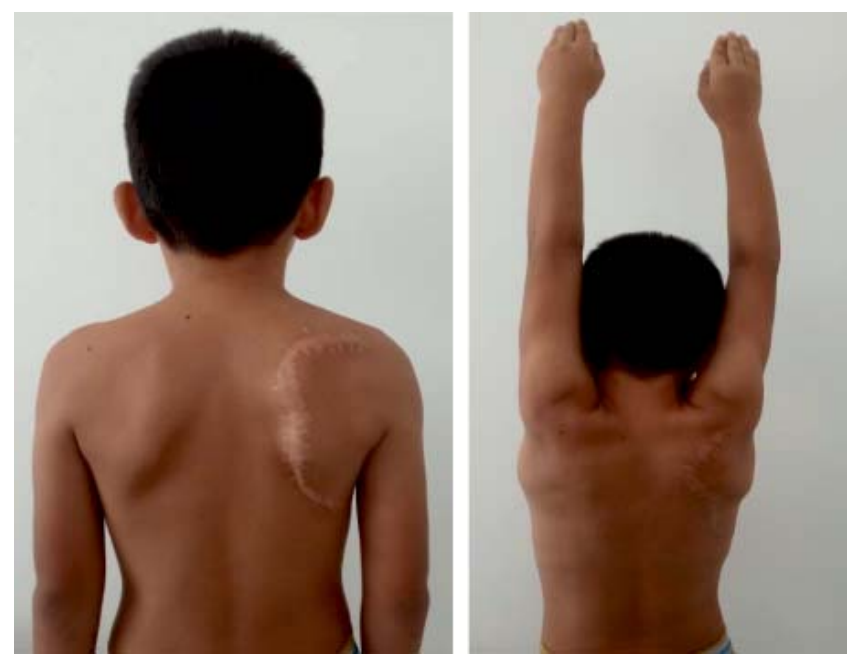

Figure 4. Condition after surgery of the same patient, after 1 year. 
Ternovsky's operation was performed to 11 (13\%) patients and Pozdeeva operation was performed to 74 (87\%) patients. After surgery, a thoracobrachial plaster cast was put on patients for 4 weeks. In addition, exercise therapy was prescribed on the fingers and UHF through a plaster cast.

After removing of plaster cast, the massage, electrophoresis with potassium iodide, alternating novocaine, exercise therapy, active sports (swimming basketball and volleyball) were prescribed.

\section{FINDINGS}

The effectiveness of the treatment was evaluated as follows:

The cosmetic result of the treatment, while maintaining the normal anatomical relations between the scapula and spine, was good in 73 sick children, as among these patients, the mild degree was 9 children, the soft tissue medium was 28 children, the soft tissue heavy was 23 children and the bone medium was 13 children. A satisfactory cosmetic result with an average bone grade was 2 children and a severe bone grade was 10 children.

The functional result of treatment is assessed by the amount of limb abduction in the shoulder joint, it was good - because the function in the shoulder joint was fully restored, the lung degree was 9 children, the soft tissue average was 21 children, or the limitation of the abduction up to $160^{\circ}$ was the soft tissue average was 7 children, the soft tissue heavy was 17 children and bone average of 8 children. The result was satisfactory - with an amplitude of shoulder abduction from $120^{\circ}$ to $160^{\circ}$,

\section{References}

1. Pozdeev, A.A., (2006) Surgical treatment of children with congenital high standing of the scapula the topic of the dissertation and abstract on PhD, Pozdeev, Andrei Aleksandrovich, St. Petersburg

2. Ozsakhin, M., Uslu, M, Inanmaz, E., Okur, M., (2012) Bilateral congenital undescended shoulder blade (sprengel deformity) Am J Phys Med Rehabil, 91: p. 374. [PubMed] [Google Scholar]

3. Pellegrin, K., Kolkman, K.A., Waldemar, K.E., (2013) Sprengel deformity, which is a post-traumatic injury in an Afghan boy: case history. Mil Med, 178: p.1379-1383. [PubMed] [Google Scholar] the soft-tissue heavy was 6 children and the bone medium was 5 children, the bone-heavy was 7 . The result was unsatisfactory - shoulder abduction does not exceed $120^{\circ}$, bone medium-2, heavy bone- 3 .

Long-term results showed that out of 85 (100\%) patients, 73 (86\%) were good and 12 (14\%) - satisfactory as the treatment results were divided into cosmetic and functional. The functional result of treatment in some patients was unsatisfactory because it was accompanied by neurological, endocrine and other abnormal changes on the part of the internal organs.

The results of treatment of children with Sprengel disease, taking into account the age at which they performed the surgery according to the methods developed by the authors have been studied.

Thus, the X-ray examination made it possible to clarify the presence or absence of the feature and severity, as well as its effect on the function of the shoulder girdle.

In summary, the analysis of the results correlates with the data of domestic and foreign literature and allows us to conclude that the operation according to the Pozdeev method is atraumatic and it is the method of choice for surgical treatment of Sprengel disease in children.

Compliance with ethics requirements: The authors declare no conflict of interest regarding this article. The authors declare that all the procedures and experiments of this study respect the ethical standards in the Helsinki Declaration of 1975, as revised in 2008(5), as well as the national law. Informed consent was obtained from all the patients included in the study.

4. O'Brien S.J., Voos D.E., Nevassier A.S., Dracos M.S. (2009) Developing anatomy of the shoulder and anatomy of the shoulder joint. In the book: Rockwood K.A., Matsen F.A., editors. Shoulder. 4th ed. Philadelphia, PA: Saunders / Elsevier; p. 1-12. [Google scientist]

5. Harvey E.J., Bernstein M., Desi N.M., Saran N., Owell J.A. (2012) Sprengel Deformation: Pathogenesis and Management. J Am Acad Orthop Surt, 20: p. 177-86. [PubMed] [Google Scholar]. 
